\title{
Non-Markoffian effects of a simple nonlinear bath
}

\author{
Hanno Gassmann, * Florian Marquardt, and C. Bruder \\ Department of Physics and Astronomy, University of Basel, Klingelbergstrasse 82, CH-4056 Basel, Switzerland
}

(Received 29 April 2002; published 24 October 2002)

\begin{abstract}
We analyze a model of a nonlinear bath consisting of a single two-level system coupled to a linear bath (a classical noise force in the limit considered here). This allows us to study the effects of a nonlinear, nonMarkoffian bath in a particularly simple situation. We analyze the effects of this bath onto the dynamics of a spin by calculating the decay of the equilibrium correlator of the $z$-component of the spin. The exact results are compared with those obtained using three commonly used approximations: a Markoffian master equation for the spin dynamics, a weak-coupling approximation, and the substitution of a linear bath for the original nonlinear bath.
\end{abstract}

DOI: 10.1103/PhysRevE.66.041111

PACS number(s): 05.40.-a, 03.65.Yz

\section{INTRODUCTION}

The linear bath of oscillators plays a prominent role in discussions of dissipation and decoherence [1,2]. In the classical limit, the force fluctuations derived from that bath correspond to a Gaussian random process. Although this is a generic case (due to the central limit theorem), there are physical situations when non-Gaussian random processes are important. In this article, we examine the simplest possible quantum-mechanical bath whose fluctuations correspond to a classical telegraph noise: a single two-level system subject to a white-noise force. The effects of this nonlinear bath are analyzed by coupling it, in turn, to a spin, whose relaxational dynamics under the action of the bath is calculated.

In the literature, another type of physically relevant nonlinear bath is usually discussed: the spin bath $[3,4]$, consisting of some large number of spins which are coupled to the system under consideration. Our model system is simpler in that it contains only a single "nonlinear element," the twolevel system. Irreversibility is generated not by having a larger number of spins but by the coupling to the linear bath. Although designed as a drastically simplified model system, it may be physically relevant for situations such as charged tunneling systems [5] in the vicinity of a mesoscopic quantum-coherent device (e.g., a Cooper-pair box [6]), which lead to electrostatic potential fluctuations and which are, themselves, also subject to dissipation and decoherence by their environment. Viewed as a whole, our model consists of two coupled two-level systems, one of which is coupled to a linear bath. Of course, such systems have been studied before, both in the context of the quantum measurement problem and decoherence of coupled qubit systems. In Refs. [7-10], the model of two spins (qubits) coupled to an environment has been analyzed in detail. However, we emphasize that our perspective and the questions addressed in this article are different from these approaches, since we are interested primarily in the differences arising from substituting the nonlinear bath (in the form of the dissipative two-level system) by a linear bath (see Fig. 1). This question is relevant, since, in many physical situations where the precise

*Electronic address: Hanno.Gassmann@unibas.ch nature of the bath decohering a given system is unknown, it is simply treated as a linear bath, with some given correlation function. It is, therefore, desirable to understand in more detail the kind and magnitude of possible errors introduced by such an approximation, in cases where the coupling cannot be assumed to be very weak.

The basic strategy is to calculate the equilibrium correlator of the two-level system exactly (which can be done in the limit of infinite temperature) and to compare the results to three common approximations. One of those involves replacing the nonlinear bath by a linear bath, whose correlation function is prescribed to be the same as that of the nonlinear bath. The others are a Markoffian master equation and a weak-coupling approximation, applied to the dynamics of the spin under the influence of the bath.

The remainder of this work is organized as follows: In Sec. II, we give the model Hamiltonian. In Secs. III-VI, the four different approaches are defined by specifying the evolution equation for the density matrix in each case. For each approach, we explain how the equilibrium correlator of the $z$ component of the spin may be obtained by solving these equations. Finally, we present plots showing the numerical

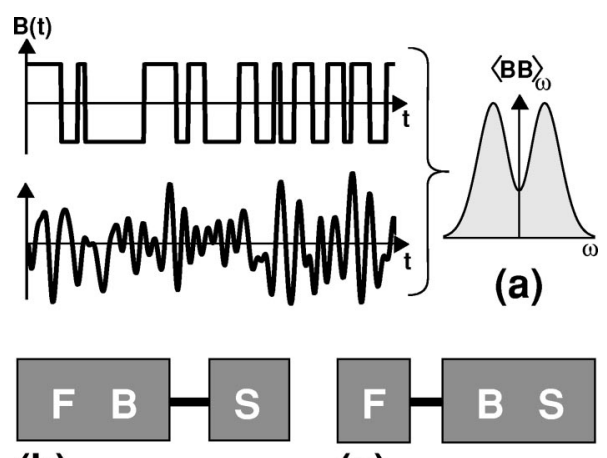

(b)

(c)

FIG. 1. (a) Schematic representation of two stochastic processes corresponding to a classical two-level fluctuator, or "telegraph noise" (top), and a Gaussian process (bottom), yielding the same power spectrum (right). (b) In our model, the two-level fluctuator $B$ is coupled to a noise force $F$ and therefore represents a (nonlinear and non-Markoffian) bath that acts on a system $S$. (c) The exact master equation description ("Approach 1" in main text) treats $S$ and $B$ as a composite system, subject to $F$. 
results for the spin correlator, along with a comparison between the different approaches.

\section{THE MODEL}

We consider a two-level system $S$ coupled to another twolevel system $B$, which represents an example of a nonlinear dissipative bath, since it is subject to a fluctuating force $F$ :

$$
\hat{H}=\epsilon_{S} \hat{\sigma}_{z}^{S}+\Delta_{S} \hat{\sigma}_{x}^{S}+J \hat{\sigma}_{z}^{S} \hat{\sigma}_{z}^{B}+\Delta \hat{\sigma}_{x}^{B}+\hat{F} \hat{\sigma}_{z}^{B}+\hat{H}_{F} .
$$

Here, the parameters $\epsilon_{S}$ and $\Delta_{S}$ serve to define any desired two-level system $S$. This system is coupled to $B$ via $\hat{\sigma}_{z}^{B}$, with the coupling strength between $S$ and $B$ being given by $J$. The oscillations of $\hat{\sigma}_{z}^{B}(t)$ at the frequency $2 \Delta$ are noisy, due to the action of the fluctuating force $\hat{F}$, which may stem from a linear bath of harmonic oscillators, whose Hamiltonian is given by $\hat{H}_{F}$. Below we will specialize to the limiting case of infinite temperature, where $\hat{F}$ becomes a purely classical noise force.

The dissipative dynamics of $S$ can be characterized in terms of several different quantities. Here we will analyze the decay of the equilibrium correlator $\left\langle\hat{\sigma}_{z}^{S}(t) \hat{\sigma}_{z}^{S}(0)\right\rangle$.

Solving the full model of a system of two interacting spins coupled to a linear bath at arbitrary temperatures and coupling strengths represents a formidable problem in itself. It has been attacked in the past using the Feynman-Vernon influence functional [11], both analytically [7] (in certain limiting cases) as well as numerically [10]. For our purposes, we will be content with analyzing a technically simpler special case. We choose $F$ to be a classical white noise fluctuating force,

$$
\langle F(t) F(0)\rangle=\gamma \delta(t)
$$

which corresponds to the limit of infinite temperature $T$ of the bath (taken such that the overall noise strength remains constant).

Under these circumstances, the dissipative dynamics of $S+B$ under the action of $F$ can be described exactly by using a Markoffian master equation. Note that this is, of course, unrelated to the validity of a master equation description for the action of $B$ onto $S$ alone, which we will discuss below. The limit of infinite temperature is dictated mostly by the desire to have a comparatively strong decay of the correlator of $\hat{\sigma}_{z}^{B}(t)$ (with a decay rate on the order of the transition frequency $2 \Delta$ of $B$ ) while still retaining the validity of a simple Markoffian master equation description (for the full system $S+B$ ). The concept of generating colored noise by coupling to a degree of freedom subject to white noise is also employed in classical stochastic mechanics, see Refs. $[12,13]$.

In the following, we will call the exact solution "Approach 1," while "Approach 2" refers to a simple master equation applied to $S$ alone, "Approach 3" replaces the nonlinear by a linear bath, and "Approach 4" is the weakcoupling approximation.

\section{THE EXACT SOLUTION: APPROACH 1}

Derivation of the master equation. First we derive the exact master equation description, which is used for the action of $F$ onto the combined system $B+S$ :

$$
\dot{\hat{\rho}}_{S B}(t)=-i\left[\hat{H}_{S B}, \hat{\rho}_{S B}(t)\right]-\gamma \hat{\rho}_{S B}(t)+\gamma \hat{\sigma}_{z}^{B} \hat{\rho}_{S B}(t) \hat{\sigma}_{z}^{B} .
$$

$\hat{H}_{S B}$ is the Hamiltonian for the system $B+S$ alone. We start with the von Neumann equation

$$
i \dot{\hat{\rho}}_{S B}^{(F)}(t)=\left[\hat{H}(t), \hat{\rho}_{S B}^{(F)}(t)\right]=\left[\hat{H}_{S B}+\hat{\sigma}_{z}^{B} F(t), \hat{\rho}_{S B}^{(F)}(t)\right],
$$

where $\hat{\rho}_{S B}^{(F)}(t)$ is the density matrix for one realization of the force $F(t) . \hat{H}(t)$ is the Hamiltonian for the system $B+S$ under the action of the force $F(t)$. Rewriting Eq. (4) in integral form, we obtain

$$
\hat{\rho}_{S B}^{(F)}(t)-\hat{\rho}_{S B}^{(F)}(0)=(-i) \int_{0}^{t} d \tau\left[\hat{H}_{S B}+\hat{\sigma}_{z}^{B} F(\tau), \hat{\rho}_{S B}^{(F)}(\tau)\right] .
$$

Iterating the last equation once leads to

$$
\begin{aligned}
\dot{\hat{\rho}}_{S B}^{(F)}(t)= & (-i)\left[\hat{H}_{S B}, \hat{\rho}_{S B}^{(F)}(t)\right] \\
& +(-i)\left[\hat{\sigma}_{z}^{B} F(t), \hat{\rho}_{S B}^{(F)}(0)\right. \\
& \left.+(-i) \int_{0}^{t} d \tau\left[\hat{H}_{S B}+\hat{\sigma}_{z}^{B} F(\tau), \hat{\rho}_{S B}^{(F)}(\tau)\right]\right] .
\end{aligned}
$$

In the following step we average over $F(\cdot)$ and use $\langle F(t)\rangle$ $=0$ and $\left\langle\hat{\rho}_{S B}^{(F)}(t)\right\rangle=\hat{\rho}_{S B}(t)$. Note that for a white-noise force $F(t) \quad$ averages factorize like $\left\langle F(t) F(\tau) \hat{\rho}_{S B}^{(F)}(\tau)\right\rangle$ $=\langle F(t) F(\tau)\rangle\left\langle\hat{\rho}_{S B}^{(F)}(\tau)\right\rangle$, since $\hat{\rho}_{S B}^{(F)}(\tau)$ depends only on the previous history of $F(\cdot)$. Therefore, we find

$$
\begin{aligned}
\dot{\hat{\rho}}_{S B}(t)= & (-i)\left[\hat{H}_{S B}, \hat{\rho}_{S B}(t)\right] \\
& +(-i)^{2} \int_{0}^{t} d \tau\left[\hat{\sigma}_{z}^{B},\left[\hat{\sigma}_{z}^{B}, \hat{\rho}_{S B}(\tau)\right]\right]\langle F(t) F(\tau)\rangle .
\end{aligned}
$$

This leads to Eq. (3) by using Eq. (2). Note that, in contrast to the usual master equation, no secular approximation [14] has been used in deriving this equation, which means the resulting decay rate does not have to be small when compared to the transition frequencies of the system $S+B$. This is possible because the bath correlation function is a delta function, which also makes the equation exact. We remark further that Eq. (3) is solved directly in the basis in which $\hat{\sigma}_{z}^{B}$ and $\hat{\sigma}_{z}^{S}$ are diagonal. A transformation into the interaction picture (as is commonly performed for the usual master equation description) would lead to explicitly timedependent terms in this equation. 
Decay of the equilibrium correlator. We want to obtain the equilibrium correlator of $\hat{\sigma}_{z}^{S}(t)$,

$$
\left\langle\hat{\sigma}_{z}^{S}(t) \hat{\sigma}_{z}^{S}(0)\right\rangle \equiv \operatorname{tr}\left[\hat{\rho}_{S B}^{(e q)} \hat{\sigma}_{z}^{S}(t) \hat{\sigma}_{z}^{S}(0)\right] .
$$

It is convenient to rewrite Eq. (8) in terms of the projector onto the spin-up state of $S, \quad \hat{P} \equiv|\uparrow\rangle_{S}\left\langle\left.\uparrow\right|_{S}=\frac{1}{2}\left(1+\hat{\sigma}_{z}^{S}\right)\right.$ :

$$
\left\langle\hat{\sigma}_{z}^{S}(t) \hat{\sigma}_{z}^{S}(0)\right\rangle=4\langle\hat{P}(t) \hat{P}(0)\rangle-1 .
$$

Here, we have used $\hat{\rho}_{S B}^{(e q)}=\frac{1}{4}$. The correlator of $\hat{P}(t)$ can be found by calculating the probability to find the system $S$ in the state "up" at the time $t$, if it had been "up" at time 0 . This has to be averaged over all realizations of the random process $F(\cdot)$ :

$$
\langle\hat{P}(t) \hat{P}(0)\rangle=\frac{1}{2} \operatorname{tr}_{B}\left\langle\left\langle\left.\uparrow\right|_{S} \hat{U}_{F}(t) \hat{P} \otimes \hat{\rho}_{B}^{(e q)} \hat{U}_{F}^{\dagger}(t) \mid \uparrow\right\rangle_{S}\right\rangle_{F} .
$$

Here $\hat{U}_{F}(t)$ is the time-evolution operator for $S+B$ under the action of a given realization of $F(\cdot)$. This equation is valid only because, in the limit of infinite temperature considered here, the probability of finding "spin up" at a certain instant of time is independent of the history of $F(\cdot)$. The expression (10) is nothing but the population $\rho_{S 11}(t)$ of the state $|\uparrow\rangle_{S}$ for a time-evolution starting from the initial condition of "spin up," $\hat{\rho}_{S B}(0)=\hat{P} \otimes \hat{\rho}_{B}^{(e q)}$ :

$$
\langle\hat{P}(t) \hat{P}(0)\rangle=\frac{1}{2} \rho_{S 11}(|t|) .
$$

Note that $\rho_{S 11}$ decays towards $1 / 2$, such that $\left\langle\hat{\sigma}_{z}^{S}(t) \hat{\sigma}_{z}^{S}(0)\right\rangle$ vanishes for $t \rightarrow \infty$ (as it should be). We have used the fact that the correlator is symmetric in time, since the potentially antisymmetric imaginary part vanishes (again due to the limit of infinite temperature). $\hat{\rho}_{S}(t)$ can be calculated by applying the master equation that describes the action of $F$ onto $S+B$. Put differently, Eq. (11) constitutes an example of the quantum regression theorem. Using $\hat{\rho}_{S}$, we calculate the Fourier transform of the equilibrium correlator of $\hat{\sigma}_{z}^{S}(t)$ :

$$
\begin{aligned}
K_{z z}^{S}(\omega) & \equiv \frac{1}{2 \pi} \int_{-\infty}^{+\infty} d t e^{i \omega t}\left\langle\hat{\sigma}_{z}^{S}(t) \hat{\sigma}_{z}^{S}(0)\right\rangle \\
& =\frac{1}{\pi} \int_{-\infty}^{+\infty} d t e^{i \omega t}\left(\rho_{S 11}(|t|)-1 / 2\right) .
\end{aligned}
$$

$K_{z z}^{S}(\omega)$ is real valued, symmetric and the integral over all frequencies gives 1 .

Time evolution of the density matrix. The master equation (3) for the density matrix $\hat{\rho}$ ( $\equiv \hat{\rho}_{S B}$ ) in the four-dimensional Hilbert space of $S+B$ represents a system of linear differential equations with constant coefficients. The latter are given by a complex-valued $16 \times 16$ matrix $C$ that corresponds to the "superoperator" on the right-hand side of the master equation. The solution is the complex vector $\rho$, which consists of the 16 components of the density matrix $\hat{\rho}$ :

$$
\dot{\rho}=-C \rho
$$

The entries of $C$ can be read off directly from Eq. (3). The formal solution of Eq. (13),

$$
\rho(t)=e^{-C t} \rho(0)
$$

can be expressed in terms of the right eigenvectors $\left|\rho^{(j)}\right\rangle$, the left eigenvectors $\left\langle\rho^{(j)}\right|$ and the eigenvalues $\lambda^{(j)}$ of $C$ :

$$
\rho(t)=\sum_{j}\left|\rho^{(j)}\right\rangle\left\langle\rho^{(j)} \mid \rho(0)\right\rangle e^{-\lambda^{(j)} t}
$$

$C$ is not necessarily Hermitian, so that the $\lambda^{(j)}$ usually are complex valued (with nonnegative real parts) and the $\left|\rho^{(j)}\right\rangle$ do not form an orthonormal basis (however, $\left\langle\rho^{(i)} \mid \rho^{(j)}\right\rangle=\delta_{i j}$ by construction). In order to obtain $\rho_{S 11}(t)$, we have to perform the trace over $B, \rho_{S 11}(t)=\rho_{S B 1111}(t)+\rho_{S B 1212}(t)$. (In $\rho_{S B s^{\prime} b^{\prime} s b}$ the indices $s, s^{\prime}$ refer to $S$, while $b, b^{\prime}$ refer to $B$.) We will use the same notation for the components of $\rho^{(j)}$, which is a complex vector. Then we obtain

$$
\int_{0}^{\infty} d t e^{i \omega t} \rho_{S 11}(t)=\sum_{j}\left(\rho_{1111}^{(j)}+\rho_{1212}^{(j)}\right) \frac{\left\langle\rho^{(j)} \mid \rho(0)\right\rangle}{\lambda^{(j)}-i \omega} .
$$

Taking the real part of this expression gives $K_{z z}^{S}(\omega)$, see Eq. (12).

Numerical results. The following steps have been performed in order to calculate the correlator $K_{z z}^{S}(\omega)$ of $\hat{\sigma}_{z}^{S}(t)$ : The entries of the matrix $C$ are obtained from Eq. (3). The eigenvalues and eigenvectors of $C$ are calculated numerically and used to get $K_{z z}^{S}(\omega)$ according to Eqs. (16) and (12).

The relevant parameters in our model are $\epsilon_{S}, \Delta_{S}, \Delta$, the coupling strength $J$, and the strength $\gamma$ of the noise force $F$. We choose the time scale such that $\Delta \equiv 1$. The results discussed in the following have been calculated for $\Delta_{S}$ $=1.2$ ( $S$ and $B$ "almost in resonance").

To begin our discussion, we note some generic features of the results obtained for Approaches 1 and 2. Since in these cases $K_{z z}^{S}(\omega)$ is essentially the Fourier transform of a density matrix relaxing according to a master equation, it consists of several Lorentzian peaks. Their number is constrained to be less than the maximum number of transition frequencies of the respective system (6 for $S+B$ in Approach 1, and 1 for $S$ in Approach 2, plus possible zero-frequency "pure" relaxation). In practice, degeneracies between transition frequencies and selection rules reduce that number to 2 (or 3 ) for Approach 1, and 1 (or 2) for Approach 2, for $\epsilon_{S}=0$ (or $\epsilon_{S}$ $\neq 0$ ).

In the limit of weak coupling, $J \rightarrow 0$, all that remains is a broadened peak at the transition frequency $2 \Delta_{S}$ of system $S$ alone. In that limit, the results for all approaches coincide, as expected (see Figs. 4 and 5). With increasing $J$, the peaks get broadened and shifted, and additional peaks may appear (in the case of Approaches 1, 3, and 4). 


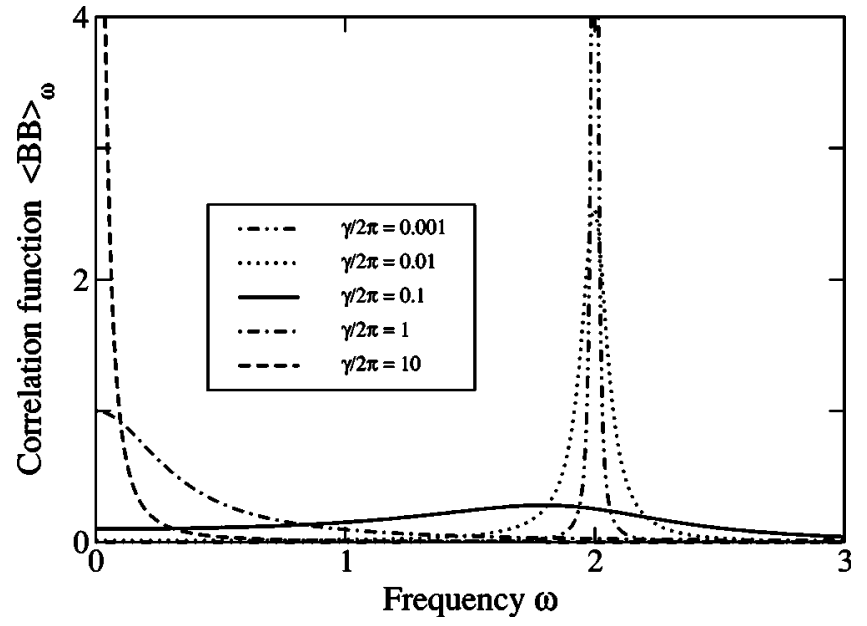

FIG. 2. The $\langle B B\rangle_{\omega}$ correlation function for different values of $\gamma$. The parameters are $J=1, \Delta=1$.

The most notable difference to the simple master equation of Approach 2 is the appearance of a second peak at the transition frequency $2 \Delta$ of the two-level fluctuator $B$. At small $J$, the strength of this peak grows like $J^{2}$, while its width is fixed (depending on $\gamma$ ). In this way, the power spectrum of the bath fluctuations shows up in the short-time behavior of the correlator of the system $S$. This behavior cannot be captured by the master equation (Approach 2).

Increasing $J$ leads to a frequency shift and a change in the width of the "original" peak at $2 \Delta_{S}$, much like predicted by the simpler Approach 2. However, in the description of the exact Approach 1, these changes are due to the change in eigenfrequencies and eigenvectors of the combined system $S+B$. At small $J$, the results of Approaches 1 and 2 can be shown to coincide using perturbation theory. Deviations from Approach 2 appear at higher values of $J$, where the energy shift of Approach 1 only grows linearly with $J$ (see Figs. 4 and 5, lower graphs). In contrast, the frequency of the second

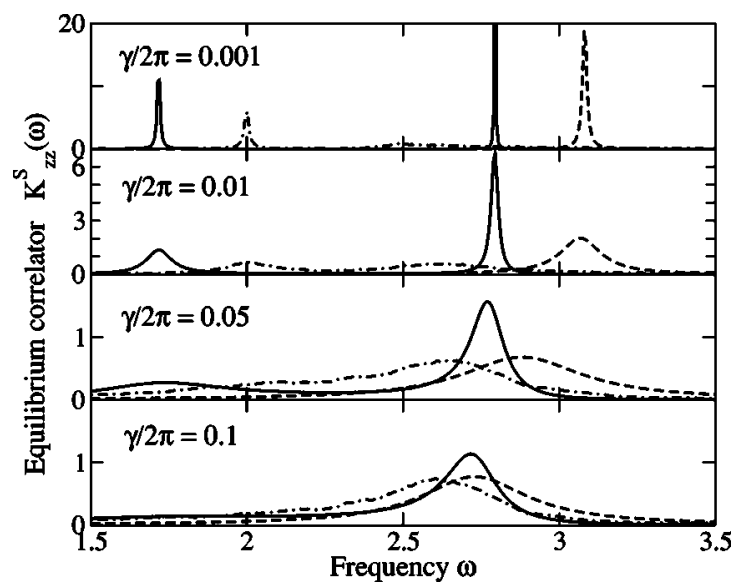

FIG. 3. The Fourier-transform $K_{z z}^{S}(\omega)$ of the equilibrium correlator of $\hat{\sigma}_{z}^{S}(t)$, for different values of the noise strength $\gamma /$ $(2 \pi)(=0.001,0.01,0.05$, and 0.1 , from topmost to lowest graph). The values of the other parameters are $\Delta=1, \Delta_{S}=1.2, J=0.5$, and $\epsilon_{S}=0$. Approaches 1 and 4, solid line; Approach 2, dashed line; Approach 3, dash-dotted line.

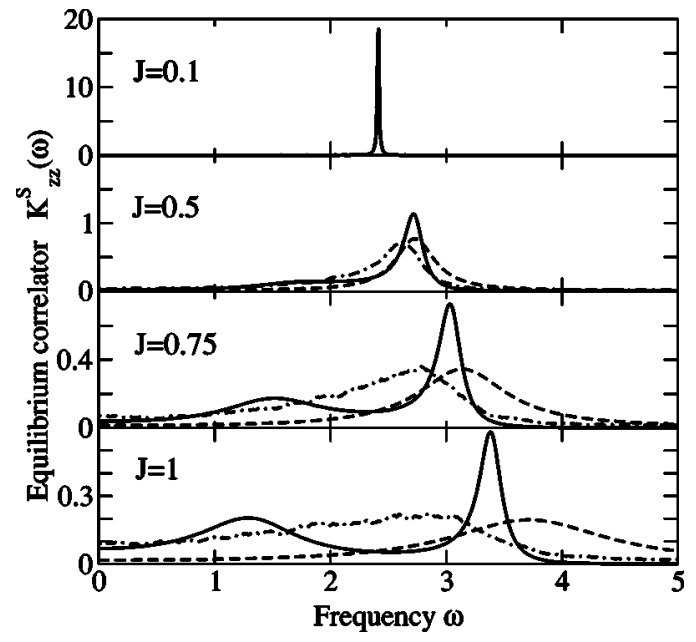

FIG. 4. The Fourier-transform $K_{z z}^{S}(\omega)$ of the equilibrium correlator of $\hat{\sigma}_{z}^{S}(t)$, for different values of the coupling strength $J$ $(=0.1,0.5,0.75$, and 1.0 , from topmost to lowest curve). The values of the other parameters are $\Delta=1, \Delta_{S}=1.2, \gamma / 2 \pi=0.1$, and $\epsilon_{S}=0$. Approaches 1 and 4, solid line; Approach 2, dashed line; Approach 3, dash-dotted line.

peak is suppressed to zero. This behavior can easily be found from the diagonalization of the Hamiltonian for the combined system $B+S$ in the limit $J \rightarrow \infty$, when one obtains two pairs of degenerate energy levels, separated by $2 J$.

Regarding the dependence on the noise strength $\gamma$, the same qualitative remarks apply as for Approach 2 (see discussion below in Sec. IV). However, it is interesting to note that there $i$ a frequency shift with increasing $\gamma$ in Approach 1 as well (see Fig. 3), in spite of the fact that the additional terms in the nonsecular master equation (3) seem to describe a purely relaxational dynamics. This is in contrast to the behavior known from the usual form of the master equation,

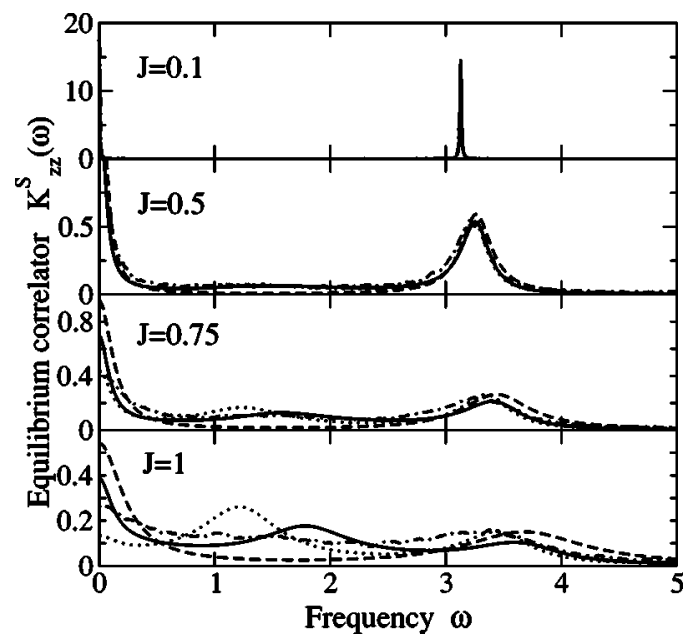

FIG. 5. The Fourier-transform $K_{z z}^{S}(\omega)$ of the equilibrium correlator of $\hat{\sigma}_{z}^{S}(t)$, for different values of the coupling strength $J$ $(=0.1,0.5,0.75$, and 1.0 , from topmost to lowest curve). The values of the other parameters are $\Delta=1, \Delta_{S}=1.2, \gamma / 2 \pi=0.1$, and $\epsilon_{S}=1$. Approach 1, solid line; Approach 2, dashed line; Approach 3, dash-dotted line; Approach 4, dotted line. 
Eq. (17), where the energy shifts can be read off directly from the imaginary coefficients in the equation.

\section{THE SIMPLE MASTER EQUATION: APPROACH 2}

As has been explained above, we will use the master equation description not only for the action of $F$ onto the combined system $S+B$ [see Eq. (3)], but also for the action of $F+B$ onto $S$ alone. This constitutes the approximate Approach 2 , involving the usual kind of master equation, which is valid only for sufficiently weak coupling $\mathrm{J}$, since it is derived by applying both the Markoff and secular approximation [see Refs. [14,15]]. In the unperturbed eigenbasis of system $S$, it reads

$$
\begin{aligned}
\dot{\rho}_{S k j}= & -\left[\Gamma_{k}+\Gamma_{j}+\widetilde{\Gamma}_{k j}+i\left(\Delta_{k}-\Delta_{j}\right)+i\left(E_{k}-E_{j}\right)\right] \rho_{S k j} \\
& +\delta_{k j} \sum_{l \neq k} \rho_{S l l}\left|A_{k l}\right|^{2} 2 \pi\langle B B\rangle_{E_{l}-E_{k}} .
\end{aligned}
$$

Equation (17) describes the relaxation of the reduced density matrix $\hat{\rho}_{S}$ of system $S$ alone, under the action of the coupling $J \hat{\sigma}_{z}^{S} \hat{\sigma}_{z}^{B}$ to the bath $B+F$. We have introduced the abbreviation $\hat{A} \equiv \hat{\sigma}_{z}^{S}$.

The Fourier transform of the correlator of $\hat{B} \equiv J \hat{\sigma}_{z}^{B}$ defines the "bath spectrum"

$$
\begin{gathered}
\langle B(t) B(0)\rangle \equiv\langle\hat{B}(t) \hat{B}(0)\rangle=J^{2}\left\langle\hat{\sigma}_{z}^{B}(t) \hat{\sigma}_{z}^{B}(0)\right\rangle, \\
\langle B B\rangle_{\omega} \equiv \frac{1}{2 \pi} \int_{-\infty}^{+\infty} d t e^{i \omega t}\langle B(t) B(0)\rangle .
\end{gathered}
$$

It is real and symmetric in the limit of infinite temperature considered here, and therefore it is equivalent to a classical colored noise force. As will be explained below, $\langle B B\rangle_{\omega}$ is found by applying the master equation (3) to $B$ alone. The decay rates are defined by

$$
\begin{gathered}
\Gamma_{k} \equiv \pi \sum_{n}\left|A_{k n}\right|^{2}\langle B B\rangle_{E_{k}-E_{n}}, \\
\widetilde{\Gamma}_{k j} \equiv-2 \pi A_{k k} A_{j j}\langle B B\rangle_{0},
\end{gathered}
$$

and the energy shifts are given via

$$
\Delta_{k} \equiv \sum_{n}\left|A_{k n}\right|^{2} \int d \omega \frac{\langle B B\rangle_{\omega}}{E_{k}-E_{n}-\omega} .
$$

Here the indices and energies refer to the unperturbed eigenstates of the original Hamiltonian of $S$ alone: $\hat{H}_{S}$ $\equiv \epsilon_{S} \hat{\sigma}_{z}^{S}+\Delta_{S} \hat{\sigma}_{x}^{S}$. The integrals should be understood as principal value integrals.

The time-evolution of $\hat{\rho}_{S}$ is found from Eq. (17) using the same approach as in Sec. III, involving the diagonalization of a superoperator $C$. In the present case, $C$ corresponds to the $4 \times 4$ matrix whose entries are read off from Eq. (17). Therefore, the equation corresponding to Eq. (16) only contains $\rho_{11}^{(j)}$, instead of the sum inside the brackets.
Furthermore, we have to obtain the equilibrium correlator of $\hat{\sigma}_{z}^{B}(t)$, which is needed as input for the master equation describing the relaxation of $S$ alone (Approach 2), the numerical sampling of random processes (Approach 3 ) and the weak-coupling approximation (Approach 4).

This is done by calculating the relaxation of $\hat{\rho}_{B}(t)$ under the action of $F$, starting from the initial condition $\hat{\rho}_{B}(0)$ $=|\uparrow\rangle_{B}\left\langle\left.\uparrow\right|_{B}\right.$ and applying the same formulas as above (with $B$ instead of $S$ ), for the master equation (3), adapted to the two-dimensional Hilbert space of $B$ (with a $4 \times 4$ matrix $C$ ). $\left\langle\hat{\sigma}_{z}^{B}(t) \hat{\sigma}_{z}^{B}(0)\right\rangle$ undergoes damped oscillations. Its Fourier transform

$$
K_{z z}^{B}(\omega)=\frac{\langle B B\rangle_{\omega}}{J^{2}}=\frac{8 \Delta^{2} \gamma}{\pi} \frac{1}{\left(\omega^{2}-4 \Delta^{2}\right)^{2}+4 \omega^{2} \gamma^{2}}
$$

consists of broad peaks of width $\gamma$ (for $\gamma^{2}<4 \Delta^{2}$ ), which is proportional to the strength of the noise force $F$ and may be comparable to the transition frequency $2 \Delta$ itself (see Fig. 2). Thus, $B$ indeed represents a noisy two-level fluctuator, which acts onto $S$ as a nonlinear (non-Gaussian) and nonMarkoffian (colored) bath.

Numerical results. First the action of $F$ onto $B$ is considered, to obtain the correlation function $\langle B B\rangle_{\omega}$. This result is given in Eq. (21). It is used to set up the master equation describing the action of $F+B$ onto $S$, Eq. (17). Its coefficients define a $4 \times 4$ " $C$ matrix," which is diagonalized. The results are inserted into the appropriately modified Eq. (16), in order to obtain $K_{z z}^{S}(\omega)$.

Naturally, the behavior of Approach 2 is simplest to analyze, since it is the textbook example of a master equation applied to a single two-level system. Since the correlator $\langle B B\rangle_{\omega}$ is proportional to $J^{2}$, both the shift of the transition frequency and the width of the peak(s) increase like $J^{2}$, for arbitrarily large $J$. In contrast, the dependence of the peak width and the frequency shift on the noise strength $\gamma$ is nonmonotonous. It is determined by the evolution of $\langle B B\rangle_{\omega}$ [see Eq. (21) and Fig. 2] with increasing $\gamma$. For very small $\gamma$, the two-level fluctuator $B$ performs very weakly damped oscillations at the frequency $2 \Delta$. Unless it is exactly at resonance with the system $S$, the dissipative effects of $B$ on the dynamics of $S$ will be weak in that regime. The decay rate of $S$, which is given by the power spectrum of $B$ evaluated at $2 \Delta_{S}$, grows linearly in $\gamma\left[\right.$ for $\gamma^{2} \ll\left(\Delta_{S}^{2}-\Delta^{2}\right)^{2} / \Delta_{S}^{2}$ ]. The transition frequency of $S$ is shifted upwards or downwards, depending on whether the main weight of the spectrum of $B$ is located below or above $\Delta_{S}\left(\Delta<\Delta_{S}\right.$ or $\left.\Delta>\Delta_{S}\right)$. For increasing $\gamma, B$ performs more strongly damped oscillations. In the limit of large $\gamma$, the spectrum $\langle B B\rangle_{\omega}$ concentrates around zero frequency (see Fig. 2) such that the decay rate of $S$ decreases again (like $1 / \gamma)$, after having gone through a maximum. The magnitude of the energy shift will also decrease for increasing $\gamma$, simply because the contributions of the power spectrum of $B$ lying to either side of $2 \Delta_{S}$ will tend to cancel each other. However, in the limit $\gamma \rightarrow \infty$, the shift always saturates at a positive value which is independent of 
$\Delta$. These facts can be read off from the analytical result for Approach 2 (written down in the special case of $\epsilon_{S}=0$ ):

$$
K_{z z}^{S}(\omega)=\frac{1}{2 \pi} \sum_{s= \pm 1} \frac{\Gamma}{\Gamma^{2}+\left(\omega-s \omega_{0}\right)^{2}} .
$$

Here the peak width is given by $\Gamma=2 J^{2} \mathfrak{R} e \Sigma\left(2 \Delta_{S}\right)$ $=2 \pi\langle B B\rangle_{2 \Delta_{S}}$, the shifted transition frequency is $\omega_{0}=2 \Delta_{S}$ $-2 J^{2} \Im m \Sigma\left(2 \Delta_{S}\right)$, and we have defined $\Sigma(\omega) \equiv(2 \gamma$ $+i \omega) /\left(-\omega^{2}+4 \Delta^{2}+2 i \omega \gamma\right)$.

The simple master equation is expected to come close to the true result, as long as the conditions of the Markoff and secular approximation are fulfilled. This means the coupling strength $J$ has to be so small that the resulting decay of $S$ proceeds slowly compared with the transition frequency itself (secular approximation) and with the correlation time of the bath (Markoff approximation). The latter is given by $\tau_{B}$ $=1 / \gamma$ if $\gamma^{2}<4 \Delta^{2}$ and $\tau_{B}=1 /\left(\gamma-\sqrt{\gamma^{2}-4 \Delta^{2}}\right)$ if $\gamma^{2}>4 \Delta^{2}$.

\section{THE NONLINEAR BATH REPLACED BY A LINEAR BATH: APPROACH 3}

Approach 3 consists in replacing the nonlinear bath by a linear one. If the two-level fluctuator $B$ were replaced by a harmonic oscillator $[16,17]$, this procedure of substituting a linear bath with an appropriate correlation function for the combination of $F$ and $B$ would be exact. Here, it is an approximation whose reliability we want to analyze by comparison to the exact solution. In our case, the fact that the power spectrum $\langle B B\rangle_{\omega}$, given in Eq. (21), is real and symmetric means that $B$ can be treated as a classical Gaussian random process. Therefore, we have to solve a Langevin equation for the density matrix

$$
\dot{\hat{\rho}}_{S}^{(B)}(t)=-i\left[\hat{H}_{\text {stoch }}(t), \hat{\rho}_{S}^{(B)}(t)\right],
$$

with the stochastic time-dependent Hamiltonian

$$
\hat{H}_{\text {stoch }}(t)=\epsilon_{S} \hat{\sigma}_{z}^{S}+\Delta_{S} \hat{\sigma}_{x}^{S}+B(t) \hat{\sigma}_{z}^{S} .
$$

We calculate numerically the time-evolution of $\hat{\rho}_{S}^{(B)}(t)$ under the action of the stochastic time-dependent Hamiltonian defined in Eq. (24), which depends on $B(t)$. The description of open quantum systems by a stochastic Schrödinger equation has recently attracted increasing attention $[15,18]$.

The density matrix $\hat{\rho}_{S}^{(B)}(t)$ has to be averaged over a statistical sample of different field configurations $B(t)$. This sample is produced by generating the Fourier coefficients of $B$ as independent complex Gaussian random variables of appropriate variance (given by the power spectrum). The field $B(t)$ itself is obtained using a Fast Fourier Transform (FFT). After averaging, we may use

$$
\langle\hat{P}(t) \hat{P}(0)\rangle=\frac{1}{2}\left\langle\rho_{S 11}^{(B)}(|t|)\right\rangle_{B}
$$

and Eqs. (10) and (12) in order to obtain $K_{z z}^{S}(\omega)$. To this end, the Fourier transform of $\rho_{S 11}(|t|)$ is calculated numerically, using a FFT on a time grid of sufficiently small step-size $\Delta t$

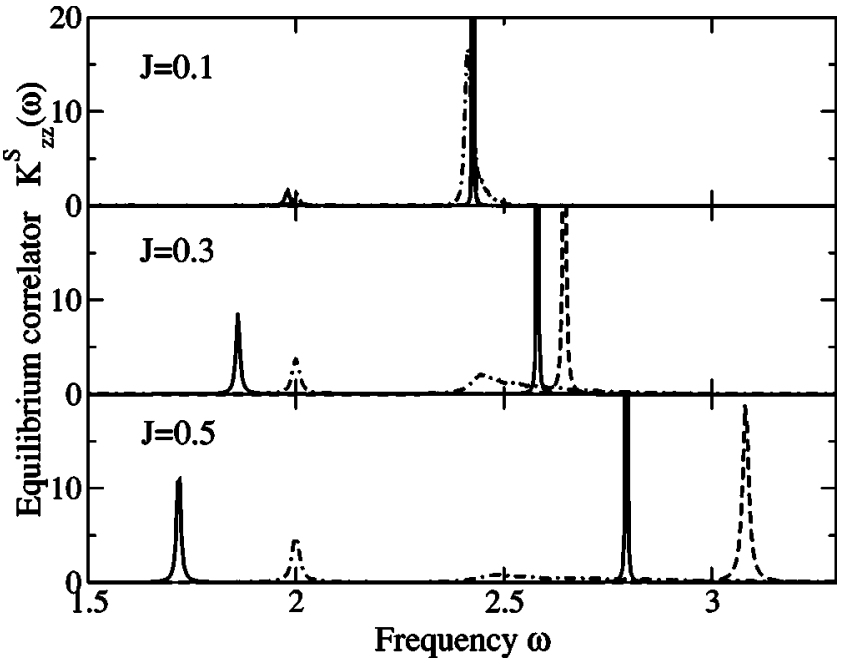

FIG. 6. The Fourier-transform $K_{z z}^{S}(\omega)$ of the equilibrium correlator of $\hat{\sigma}_{z}^{S}(t)$, for different values of the coupling strength $J$ $(=0.1,0.3$, and 0.5 ; from topmost to lowest curve). The values of the other parameters are $\Delta=1, \Delta_{S}=1.2, \gamma / 2 \pi=0.001$, and $\epsilon_{S}$ $=0$. Approaches 1 and 4, solid line; Approach 2, dashed line; Approach 3, dash-dotted line.

and sufficiently large length. The results displayed in the figures have been obtained using $10^{4}$ samples and a frequency resolution of $\Delta \omega=2 \pi / 800$. The curves have been smoothed by averaging over 5 to 20 adjacent frequency bins.

Since Approach 3 takes the full bath spectrum $\langle B B\rangle_{\omega}$ as input, this spectrum may also show up in the result for the system correlator $K_{z z}^{S}(\omega)$, as is indeed the case. Figure 3 demonstrates that this effect is most pronounced for small values of $\gamma$, where the bath spectrum has a relatively sharp structure [the noise field $B(\cdot)$ acting on $S$ deviates strongly from white noise]. In these cases, the qualitative agreement between Approach 3 and Approach 1 ("exact solution") is much better than that between Approach 2 ("simple master equation") and Approach 1 (see also Fig. 6). Nevertheless, there are deviations: In particular, there is no visible shift of the peaks in Approach 3 with increasing $J$. They just become wider and asymmetric (this applies especially to the peak at frequency $2 \Delta_{S}$ ). For higher values of $\gamma$, the linear bath (Approach 3 ) in general shows less structure than the exact solution, obtained for the actual nonlinear bath.

\section{THE WEAK-COUPLING APPROXIMATION: APPROACH 4}

Instead of the Markoff approximation one can use a weakcoupling approximation [15]. This keeps the full information contained in the correlator $\langle B B\rangle_{\omega}$, at the price of introducing a kernel for the master equation which is no longer local in time. Let us shortly describe the derivation of the weakcoupling equation. The von Neumann equation, iterated up to second order, reads in the interaction picture with respect to $\hat{H}_{0} \equiv \hat{H}-\hat{V}$ : 


$$
\dot{\hat{\rho}}_{I}(t)=-i\left[\hat{V}_{I}(t), \hat{\rho}_{I}(0)\right]-\int_{0}^{t} d \tau\left[\hat{V}_{I}(t),\left[\hat{V}_{I}(\tau), \hat{\rho}_{I}(\tau)\right]\right] .
$$

The density matrix is replaced by an approximate factorized density matrix $\hat{\rho}_{I}(t)=\hat{\rho}_{S I}(t) \otimes \hat{\rho}_{B}(0)$, where the bath remains described by a thermal equilibrium distribution. The equation becomes

$$
\begin{aligned}
\dot{\hat{\rho}}_{S I}(t)= & -i \operatorname{tr}_{B}\left[\hat{V}_{I}(t), \hat{\rho}_{S}(0) \otimes \hat{\rho}_{B}(0)\right] \\
& -\int_{0}^{t} d \tau t r_{B}\left[\hat{V}_{I}(t),\left[\hat{V}_{I}(\tau), \hat{\rho}_{S I}(\tau) \otimes \hat{\rho}_{B}(0)\right]\right],
\end{aligned}
$$

where a trace over the bath, which also includes $F$, has been taken. Now we introduce the interaction operator $\hat{V}$, which is given by $\hat{V}=\hat{A} \hat{B}$. In our case, $\hat{B}$ has zero mean and its correlator is symmetric, see Eq. (21). We get

$$
\dot{\hat{\rho}}_{S I}(t)=-\int_{0}^{t} d \tau\left[\hat{A}_{I}(t),\left[\hat{A}_{I}(t-\tau), \hat{\rho}_{S I}(t-\tau)\right]\right]\langle B(\tau) B(0)\rangle,
$$

Going back to the Schrödinger picture and inserting $\hat{A}=\hat{\sigma}_{z}^{S}$ then leads to the following weak-coupling equation, which is second order in $J$ :

$$
\begin{aligned}
\dot{\hat{\rho}}_{S}(t)= & -i\left[\hat{H}_{S}, \hat{\rho}_{S}(t)\right]-\int_{0}^{t} d \tau\left[\hat{\sigma}_{z}^{S}, e^{-i \hat{H}_{S} \tau}\right. \\
& \left.\times\left[\hat{\sigma}_{z}^{S}, \hat{\rho}_{S}(t-\tau)\right] e^{i \hat{H}_{S} \tau}\right]\langle B(\tau) B(0)\rangle .
\end{aligned}
$$

This equation is conveniently solved by using the Laplace transform. The Laplace transform of the equilibrium correlator of the bath $B$ is connected to the Fourier transform in the usual way

$$
\begin{aligned}
C_{B B}(s) & \equiv \int_{0}^{\infty} d t e^{-s t}\langle B(t) B(0)\rangle=\int_{-\infty}^{\infty} d \omega \frac{\langle B B\rangle_{\omega}}{s+i \omega} \\
& =J^{2} \frac{s+2 \gamma}{s^{2}+2 \gamma s+4 \Delta^{2}} .
\end{aligned}
$$

Using the Laplace transform, the system of differential equations becomes a system of linear algebraic equations, which can be solved by matrix inversion. All the results can be obtained analytically. However, here we only present the comparatively brief expression for the special case of $\epsilon_{S}$ $=0$ :

$$
K_{z z}^{S}(\omega)=\frac{1}{\pi} \mathfrak{R e}\left\{\frac{s+4 C_{B B}(s)}{s^{2}+4 s C_{B B}(s)+4 \Delta_{S}^{2}} \mid s=-i \omega\right\} .
$$

These analytical results [see, e.g., Eq. (31)] are then evaluated with the appropriate numerical values of the parameters.
In general, we would expect the weak-coupling solution to be a bit worse than the simulation of the linear bath with colored noise correlations (Approach 3), since it is an approximation to the latter case. However, the result for the special case $\epsilon_{S}=0$ turns out to coincide completely with the exact solution (Approach 1). The second peak shows up, in contrast to the Markoff approximation. The solution for $\epsilon_{S}$ $=1$ (or, more generally, $\epsilon_{S} \neq 0$ ) is good for small systembath coupling $J$. It fails for increasing $J$, where Approach 3 seems to be the better approximation, provided $\epsilon_{S}$ is not too small (see discussion above).

\section{CONCLUSIONS}

We have discussed a simple model of a nonlinear bath, consisting of a single two-level system subject to a classical white-noise force. Its action on another two-level system has been analyzed using four different approaches. Numerical results for various special cases have been obtained and discussed. The regimes where the different approaches work well became clear: The standard Markoff approximation gives good results as long as the coupling strength is so small that the decay is slow compared to the transition frequency and the bath correlation time. However, if the bath spectrum displays sharp structures, their effects on the system's correlator are only retained in the weak-coupling equation with its memory kernel. As expected, both approaches fail in the regime of large coupling between system and bath. In that regime the linear bath may still provide a good approximation to the original nonlinear bath. Again, this applies in particular when the bath spectrum has a strongly peaked structure. However, deviations between the linear and the original nonlinear bath are clearly visible. Although we only discussed the simplest example of a nonlinear bath, we expect the statements about the regimes where the different approximations work to be valid also for more complicated systems.

From the point of view of the computational effort, the stochastic simulation used in Approach 3 is the most unfavorable one, because the differential equations have to be integrated numerically many times to get the statistical average. The master equation and the weak-coupling approximation require the least effort, in particular because explicit analytical expressions could be found for our model.

We plan to adress the (technically more involved) case of arbitrary finite temperatures in the future. Further possible extensions include an analysis of the higher-order terms in the weak-coupling equation, as well as replacing the twolevel system $B$ by a spin of larger magnitude, to observe the transition to the linear bath.

\section{ACKNOWLEDGMENTS}

We like to thank H.-A. Engel, G.-L. Ingold, D. Loss, P. Talkner, W. Zwerger, and particularly F. Meier for helpful discussions. Our work was supported by the Swiss NSF and the NCCR Nanoscience. 
[1] A. O. Caldeira and A. J. Leggett, Ann. Phys. (N.Y.) 140, 374 (1983); Physica A 121, 587 (1983).

[2] U. Weiss, Quantum Dissipative Systems (World Scientific, Singapore, 2000).

[3] N. Prokof'ev and P. Stamp, Rep. Prog. Phys. 63, 669 (2000).

[4] A. V. Khaetskii, D. Loss, and L. Glazman, Phys. Rev. Lett. 88, 186802 (2002).

[5] E. Paladino, L. Faoro, G. Falci, and R. Fazio, Phys. Rev. Lett. 88, 228304 (2002).

[6] Y. Nakamura, Yu. A. Pashkin, and J. S. Tsai, Nature (London) 398, 786 (1999).

[7] M. Dube and P. C. E. Stamp, Chem. Phys. 268, 257 (2001).

[8] D. Loss and D. P. DiVincenzo, Phys. Rev. A 57, 120 (1998).

[9] M. Governale, M. Grifoni, and G. Schön, Chem. Phys. 268, 273 (2001).

[10] M. Thorwart and P. Hänggi, Phys. Rev. A 65, 012309 (2002).
[11] R. P. Feynman and F. L. Vernon, Ann. Phys. (N.Y.) 24, 118 (1963).

[12] N. P. van Kampen, Stochastic Processes in Physics and Chemistry (North-Holland, New York, 1992).

[13] H. Risken, The Fokker-Planck Equation (Springer, Heidelberg, 1989).

[14] K. Blum, Density Matrix Theory and Applications (Plenum Press, New York, 1996).

[15] C. W. Gardiner and P. Zoller, Quantum Noise (Springer, Heidelberg, 1991).

[16] A. Garg, J. N. Onuchic, and V. Ambegaokar, J. Chem. Phys. 83, 4491 (1985).

[17] F. K. Wilhelm (unpublished).

[18] J. T. Stockburger and H. Grabert, Phys. Rev. Lett. 88, 170407 (2002). 